\title{
Clinical significance of CXCL16/CXCR6 expression in patients with prostate cancer
}

\author{
HONG KOO HA, WAN LEE, HYUN JUN PARK, SANG DON LEE, JEONG ZOO LEE and MOON KEE CHUNG \\ Department of Urology and Medical Research Institute, Pusan National University Hospital, \\ Pusan National University School of Medicine, Busan 602739, Republic of Korea
}

Received October 15, 2010; Accepted February 17, 2011

DOI: $10.3892 / \mathrm{mmr} .2011 .446$

\begin{abstract}
We hypothesized that the CXCL16-CXCR6 ligandreceptor system may play an important role in prostate cancer progression. Levels of CXCL16 and CXCR6 expression were evaluated in prostate cancer cell lines (PC-3 and LNCaP) and normal prostate epithelial cells (PrEC), as well as in tissues from 354 patients. The immunohistochemical expression of CXCL16/ CXCR6 was greater in the PC-3/LNCaP cells than in the PrEC cell line. The expression of CXCL16/CXCR6 was significantly higher in prostate cancer than in benign prostatic hypertrophy. Using RT-PCR, the expression of CXCL16/CXCR6 was found to be greater in the PC-3/LNCaP cells than in the PrEC cell line. CXCL16/CXCR6 was weakly detected in lung and liver tissues, whereas CXCL16 was highly expressed in specimens of bone metastasis. CXCL16 immunostaining was related to Gleason score, $\mathrm{T}$ stage, tumor volume, perineural invasion and lymph node metastasis. However, biochemical PSA recurrence was not related to the expression of CXCL16/CXCR6. High CXCL16/ CXCR6 expression may be related to aggressive cancer behavior, and high CXCL16 expression to bone metastases.
\end{abstract}

\section{Introduction}

Prostate cancer (PCa) accounts for $4.2 \%$ of all cancer cases in Korea, and its morbidity has increased sharply in recent years (1). Although the incidence of localized PCa has increased due to early screening prostate-specific antigen (PSA) tests, cases of locally advanced and metastatic PCa are still frequently encountered. The high mortality rate of $\mathrm{PCa}$ is closely related to the spread of malignant prostate cells to other organs (2). To improve the diagnosis and therapy of localized and metastatic $\mathrm{PCa}$, the identification of molecules involved in the development and progression of this disease is a high priority.

Correspondence to: Dr Hong Koo Ha, Department of Urology and Medical Research Institute, Pusan National University Hospital, Pusan National University School of Medicine, 305 Gudeok-ro, Seo-ku, Busan 602739, Republic of Korea

E-mail: hongkooha@naver.com; hongkooha@pusan.ac.kr

Key words: CXCL16 protein, CXCR6 protein, neoplasm metastasis, prostatic neoplasm
Chemokines are proinflammatory chemoattractant cytokines that function primarily to direct the migration of various types of leukocytes through interactions with a group of seven-transmembrane-stretch $\mathrm{G}$ protein-coupled receptors (2). To date, over 50 chemokines and 20 chemokine receptors have been identified. These are grouped into four categories, $\mathrm{C}, \mathrm{CC}, \mathrm{CXC}$ and $\mathrm{CX} 3 \mathrm{C}$, according to the location of the main cysteine residues near the $\mathrm{N}$ terminal of these proteins (3). CXCL16 is one of the two known transmembrane chemokines. It is not only found on immune cells, but is also constitutively expressed on fibroblasts, keratinocytes and cancer cells of various origins (4-6). Recently, CXCL16 was identified as a ligand for CXCR6, which is expressed by peripheral blood leukocytes (7). Hu et al found that CXCR6 was expressed in human $\mathrm{PCa}$, and that this protein was associated with the invasion of PC-3 and LNCaP cells in vitro (8). However, few studies have examined CXCR6 expression in PCa cell lines.

To investigate whether the CXCR6/CXCL16 ligandreceptor system is involved in the progression of human $\mathrm{PCa}$, we investigated the expression of CXCL16 and CXCR6 protein in the PCa cell lines PC-3 and LNCaP and compared it to that in normal prostate epithelial cells (PrEC). Additionally, we performed immunohistochemical (IHC) staining for CXCR6/CXCL16 and quantitative real-time reverse-transcriptase polymerase chain reaction (RT-PCR) in clinical tissue samples of $\mathrm{PCa}$ and benign prostatic hyperplasia $(\mathrm{BPH})$, and evaluated the relationship between CXCR6/CXCL16 expression and the clinicopathological findings with reference to PCa progression.

\section{Materials and methods}

Human tissue collection and classification. PCa and $\mathrm{BPH}$ tissues were obtained from 354 patients who underwent radical prostatectomy or holmium laser enucleation of the prostate. These specimens were provided by the Korean National Biobank of PNUH. Paraffin blocks for IHC staining were available from 319 of the patients and frozen tissues for real-time RT-PCR from 26 patients. Additionally, bone, liver and lung tissues were sampled during surgery or biopsy from patients with non-malignant diseases ( 2 cases of herniated nucleus pulposus, 1 of hemangioma of the liver and 2 of pneumothorax). Metastatic lumbar bone marrow tissue was harvested from 2 patients with PCa. 
Patient age, preoperative serum prostate-specific antigen (PSA) levels, Gleason grade, stage, tumor volume, lymphovascular invasion, perineural invasion, surgical margin and pelvic lymph node metastasis were evaluated in the prostatectomy specimens. The pathological stage of $\mathrm{PCa}$ was categorized according to the 1997 International Union against Cancer and American Joint Committee on Cancer TNM classification (9). Two independent pathologists classified each sample according to the 2005 International Society of Urological Pathology modified Gleason system (10).

All procedures involving study participants were approved by the Institutional Review Board (IRB approved protocol no. PNUH-2010021). The use of tissues for research purposes was approved by the Pusan National University Hospital (PNUH) Ethics Committee under the title 'Clinical significance of CXCL16/CXCR6 interaction in patients with prostate cancer' (2010-6) on 5 February, 2010, and written consent was obtained from the donors.

Cell culture. The PC-3, LNCaP and primary PrEC cell lines (CRL-1435, CRL-1740 and PCS-440-010, respectively) were obtained from the American Type Culture Collection (ATCC; Manassas, VA, USA). The PC-3 and LNCaP cell lines were cultured in F-12K and RPMI-1640 medium (ATCC), respectively. PrECs were cultured with a PrEC basal medium and growth kit (PCS-440-030 and PCS-440-040; ATCC) according to the manufacturer's instructions, and used without passage. The $\mathrm{LNCaP}$ cells were originally isolated from a lymph node of a patient with hormone-dependent $\mathrm{PCa}$ with bone and lymph node metastases. PC-3 cells were isolated from vertebral metastases in hormone-independent $\mathrm{PCa}$ patients. The PrEC line was used as the control. All cell lines were cultured at $37^{\circ} \mathrm{C}$ in a humidified atmosphere of $5 \% \mathrm{CO}_{2}$ and passaged upon reaching $\sim 70 \%$ confluence.

Immunohistochemical staining. To confirm the expression of CXCR6 and its ligand CXCL16, IHC staining of the PC-3, $\mathrm{LNCaP}$ and PrEC cell lines and of the $35 \mathrm{BPH}$ and $319 \mathrm{PCa}$ tissue specimens was performed.

The tissues were routinely fixed with formalin and embedded in paraffin. Antigen retrieval was carried out and $0.3 \% \mathrm{H}_{2} \mathrm{O}_{2}$ in phosphate-buffered saline (PBS) was used to block endogenous peroxidase activity in the sections. After treatment with protein-blocking solution containing $7 \%$ horse serum to block non-specific binding, the sections were incubated overnight at $4^{\circ} \mathrm{C}$ with $0.5 \mu \mathrm{g} / \mathrm{ml}$ rabbit antihuman CXCR6 (Gentex Corporation, Zeeland, MI, USA) and $0.3 \mu \mathrm{g} / \mathrm{ml}$ rabbit anti-human CXCL16 (PeproTech, Rocky Hill, NJ, USA) antibodies. A streptavidin-biotin detection reagent kit (Ultravision LP detection system; Thermo Fisher Scientific, Waltham, MA, USA) with 3,3'-diaminobenzidine tetrahydrochloride was used for signal detection, and Harris hematoxylin was used as a counterstain. For negative controls, sections were stained using rabbit or biotinylated goat $\operatorname{IgG}$ (20 $\mu \mathrm{g} / \mathrm{ml}$; R\&D Systems, Abingdon, UK). Immunostaining for CXCL16 and CXCR6 was scored independently by two experienced pathologists. Immunostaining intensity was scored as: 0 , no staining; 1 , yellow staining; and 2 , brown staining, at a magnification of $x 400$. The percentage of positive cells was calculated as: $0,<5 \% ; 1,5-25 \% ; 2,25-50 \% ; 3,50-75 \%$; and 4 ,
$>75 \%$, at a magnification of $\mathrm{x} 100$. The IHC staining score was defined as the sum of the intensity and percentage scores (8).

Total RNA extraction. To confirm the expression of the mRNA transcripts of CXCR6 and its ligand CXCL16, quantitative real-time RT-PCR was performed using PC-3, LNCaP and PrEC cells as well as bone, liver, lung and metastatic bone tissues. Total RNA was extracted using QIAzol ${ }^{\mathrm{TM}}$ lysis reagent (Qiagen, Valencia, CA, USA). Briefly, the samples were minced and lysed in the QIAzol lysis reagent. Subsequently, a 1/5 volume of chloroform was added and the mixture was agitated vigorously for $15 \mathrm{sec}$ and then maintained on ice for 5 $\mathrm{min}$. The suspension was centrifuged at $12,000 \mathrm{rpm}$ at $4^{\circ} \mathrm{C}$ for $15 \mathrm{~min}$. The upper aqueous phase was transferred to a fresh tube, mixed with an equal volume of isopropanol, and then maintained on ice for $5 \mathrm{~min}$. The resulting RNA was precipitated and pelleted by centrifugation at $12,000 \mathrm{rpm}$ at $4^{\circ} \mathrm{C}$ for $15 \mathrm{~min}$. The RNA pellets were washed with $75 \%$ ethanol by vortexing and subsequent centrifugation at $12,000 \mathrm{rpm}$ at $4^{\circ} \mathrm{C}$ for $5 \mathrm{~min}$. The pellets were dried briefly and dissolved in diethyl -pyrocarbonate (DEPC)-treated RNase-free solution. Total RNA was quantified by measuring the absorbance at $260 \mathrm{~nm}$.

Reverse transcription of RNA. The prepared RNA was reverse transcribed into complementary DNA (cDNA) in a 20- $\mu 1$ volume containing 5X RT reaction buffer plus $0.005 \mathrm{M}$ DTT, $1 \mathrm{mM}$ of each dNTP, $20 \mathrm{U}$ RNase inhibitor, $50 \mu \mathrm{M}$ oligo (dT) primer, $2 \mu \mathrm{g}$ total RNA and $200 \mathrm{U}$ of DiaStar ${ }^{\mathrm{TM}}$ RTase (SolGent, Daejeon, South Korea). The mixture was then incubated at $50^{\circ} \mathrm{C}$ for $50 \mathrm{~min}$ and then at $70^{\circ} \mathrm{C}$ for $10 \mathrm{~min}$.

Quantitative real-time RT-PCR. The mRNA of CXCR6 and its ligand CXCL16 was quantified using commercial LightCycler FastStart DNA Master SYBR Green I (Roche, Basel, Switzerland) using the LightCycler instrument (Roche Molecular Diagnostics, Pleasanton, CA, USA) for real-time PCR and all subsequent quantification steps according to the manufacturer's instructions.

The PCR primer pairs used for cDNA amplification were as follows: 5'-CTGACTCAGCCAGGCAATGG-3' (sense) and 5'-TGAGTGGACTGCAAGGTGGA-3' (antisense) for human CXCL16; 5'-ATGGCAATGTCTTTAATCTCGACAA-3' (sense) and 5'-TGAAAGCTGGTCATGGCATAGTATT-3' (antisense) for human CXCR6; and 5'-GGGGAGCCAAAAGGGTCATC ATCT-3' (sense) and 5'-GAGGGGCCATCCACAGTCTTCT-3' (antisense) for human GAPDH. A typical 20- $\mu$ l one-tube PCR reaction contained $1 \mu \mathrm{l}$ cDNA (sample) or serially diluted standard cDNA. PCR amplifications were performed in separate tubes for 50 cycles $\left(10 \mathrm{sec}\right.$ at $95^{\circ} \mathrm{C} ; 5 \mathrm{sec}$ at $57^{\circ} \mathrm{C}$ and $15 \mathrm{sec}$ at $72^{\circ} \mathrm{C}$ ) using PCR Master mixtures specific for CXCR6 or CXCL16 or the GAPDH housekeeping gene. The GAPDH reaction product served as a control for PCR and as a reference for the relative quantification of CXCR6 and CXCL16 mRNA.

The number of PCR cycles required to reach the fluorescence threshold was defined as the cycle threshold $(\mathrm{Ct})$. The $\mathrm{Ct}$ value for each sample was proportional to the $\log$ of the initial amount of cDNA input. By plotting the $\mathrm{Ct}$ value of an unknown sample on a standard curve, the amount of target sequence in the sample was calculated. To normalize the CXCR6 and CXCL16 mRNA expression for sample-to-sample 


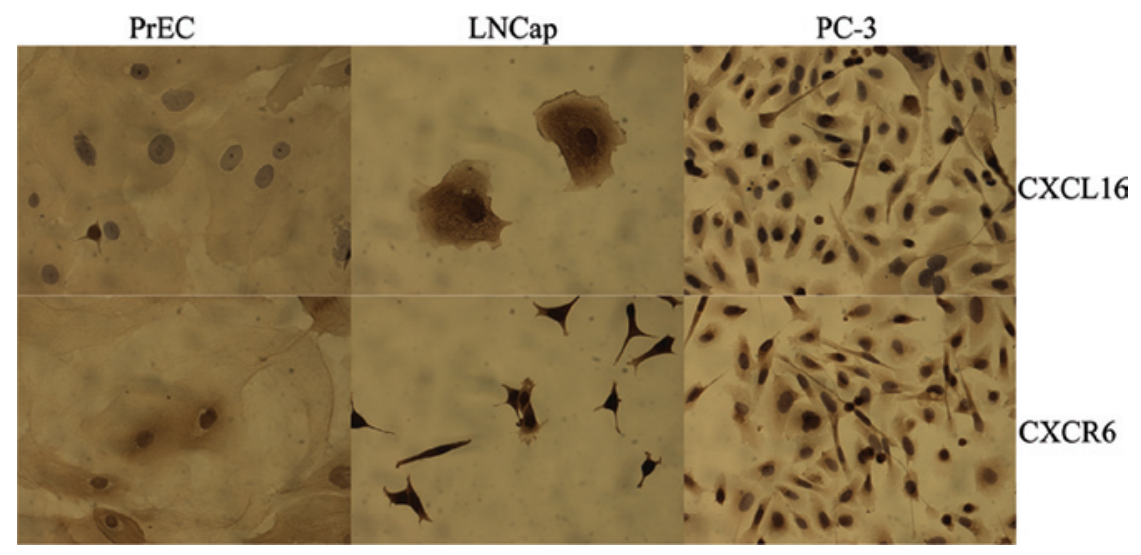

Figure 1. Immunohistochemical staining of CXCL16/CXCR6 in prostate cancer cell lines. Magnification, x400.

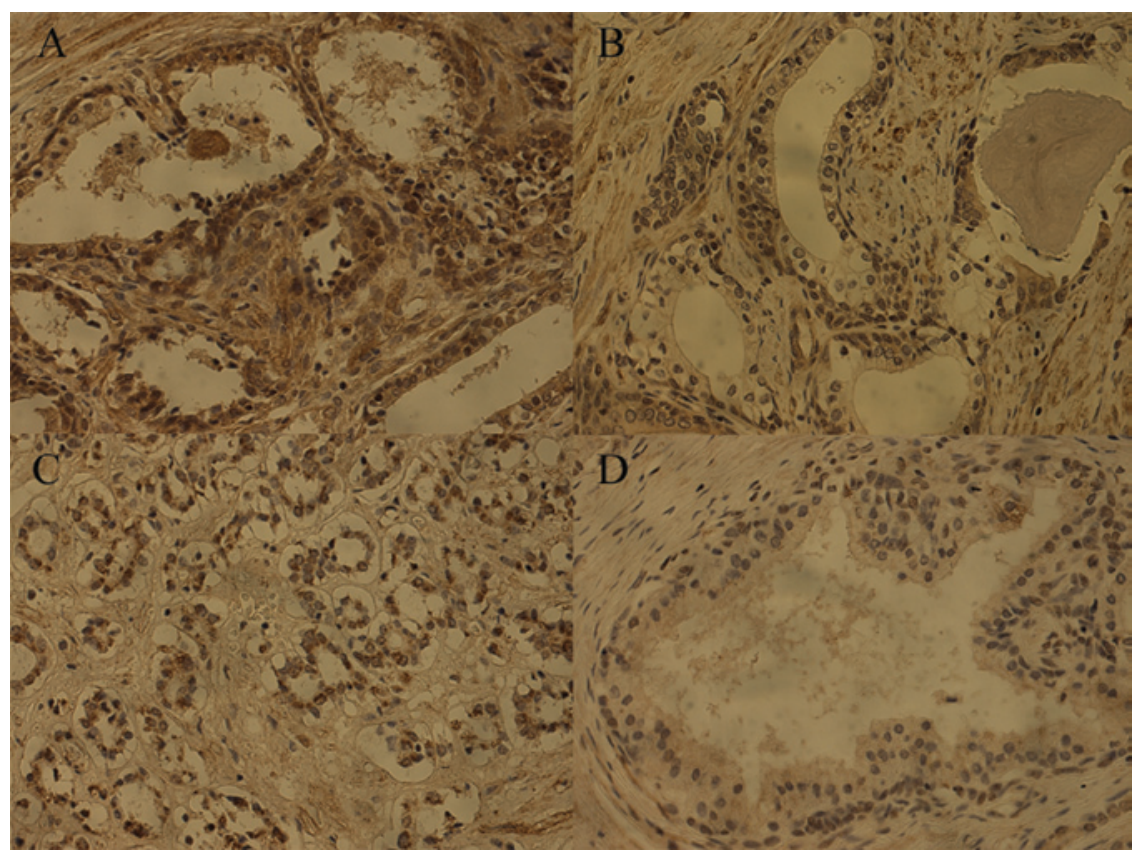

Figure 2. Immunohistochemical staining of CXCL16/CXCR6 in prostate cancer tissues (A, CXCL16; C, CXCR6; prostate-specific antigen, 12.01 ng/ml; Gleason score 3+4 and T2cN0M0) and benign prostatic hyperplasia tissues (B, CXCL16; D, CXCR6; prostate volume 48 ml; holmium laser prostatectomy). Magnification, $\mathrm{x} 400$.

differences in RNA input, RNA quality and reverse transcriptase efficiency, the GAPDH housekeeping gene was amplified. The calculated amounts of CXCR6, CXCL16 and $G A P D H$ were derived from the standard curve.

Statistical analysis. Statistical analysis was conducted using SPSS software (version 15.0 for Windows; SPSS, Chicago, IL, USA). Statistical differences in CXCR6 and CXCL16 protein expression between the $\mathrm{PCa}$ and $\mathrm{BPH}$ cells were evaluated using Pearson's Chi-square test. The Pearson coefficient was used to assess the statistical significance of differences between the molecular and clinicopathological parameters. Univariate survival analysis was performed according to Kaplan-Meier, and differences in the survival curves were assessed with the log-rank test. Multivariate analysis was performed on all of the parameters that were found to be significant through univariate analysis using the Cox regression model, with the level of significance set at $\mathrm{p}<0.05$.

\section{Results}

\section{Immunohistochemical staining}

In vitro. The PrEC cell line exhibited weak CXCR6 immuno-reactivity, while the expression of CXCR6 was increased in the PC-3 and LNCaP cells. The immunostaining scores for CXCR6 were 2.00 and 3.50 in the PrEC and PCa cell lines, respectively. The pattern of CXCL16 expression was similar to that of CXCR6, with mean values of 3.00 and 4.00, respectively (Fig. 1).

In vivo. The positive CXCL16 and CXCR6 immunoreactivity was higher in the $\mathrm{PCa}$ tissues than in the $\mathrm{BPH}$ specimens $(p=0.032$ and $p=0.079$, respectively). In the $\mathrm{PCa}$ tissues, CXCL16 immunoreactivity was positive in 272 of 319 samples (85.3\%), and CXCR6 expression was noted in 222 of 319 samples $(69.6 \%)$. In the BPH tissues, the immunoreactivity was decreased compared to the $\mathrm{PCa}$ tissues. Positive CXCL16 immunoreactivity was observed in 22 of 35 samples 
A
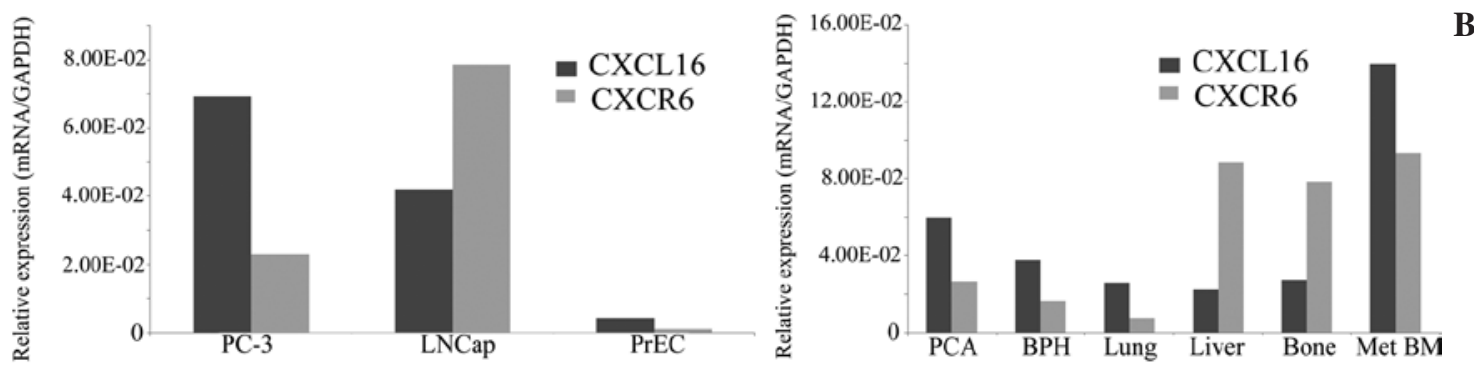

Figure 3. (A) Quantitative real-time RT-PCR of CXCR6 and CXCL16 in prostate cancer cell lines (PC-3 and LNCaP) and a prostate epithelial cell line (PrEC). The expression of CXCL16/CXCR6 was increased to a greater extent in the PC-3/LNCaP cells than in the PrEC cell line. (B) Quantitative real-time RT-PCR of CXCL16 and CXCR6 in prostate tissue, lung, liver, bone and metastatic bone marrow (Met BM). The expression of CXCL16 was highly increased in the metastatic bone tissues.

Table I. Relationship between the expression of CXCL16/CXCR6 and clinicopathologic characteristics.

\begin{tabular}{|c|c|c|c|c|c|c|}
\hline \multirow[t]{2}{*}{ Characteristic } & \multicolumn{5}{|c|}{ IHC staining } & \multirow{2}{*}{$\frac{\mathrm{BCR}}{\text { p-value (univariate analysis) }}$} \\
\hline & No. & CXCL16 & $\mathrm{p}$-value & CXCR6 & p-value & \\
\hline PSA (ng/ml) & & & 0.182 & & 0.108 & $<0.001$ \\
\hline$\leq 20$ & 182 & 3.49 & & 2.68 & & \\
\hline$>20$ & 137 & 4.02 & & 3.42 & & \\
\hline Gleason score & & & 0.042 & & 0.051 & 0.002 \\
\hline$\leq 7$ & 208 & 2.65 & & 2.57 & & \\
\hline$>7$ & 111 & 4.81 & & 3.48 & & \\
\hline T stage & & & 0.046 & & 0.185 & $<0.001$ \\
\hline$\leq \mathrm{T} 2 \mathrm{c}$ & 228 & 2.87 & & 2.72 & & \\
\hline$>\mathrm{T} 2 \mathrm{c}$ & 91 & 4.58 & & 3.38 & & \\
\hline Tumor volume & & & 0.028 & & & 0.178 \\
\hline$\leq 30 \%$ & 85 & 2.54 & & 3.27 & 0.254 & \\
\hline$>30 \%$ & 234 & 4.96 & & 2.83 & & \\
\hline Perineural invasion & & & 0.022 & & 0.068 & 0.112 \\
\hline Negative & 95 & 2.30 & & 2.69 & & \\
\hline Positive & 224 & 5.15 & & 3.40 & & \\
\hline LN metastasis & & & 0.037 & & 0.171 & $<0.001$ \\
\hline Negative & 28 & 2.37 & & 3.38 & & \\
\hline Positive & 291 & 5.08 & & 2.72 & & \\
\hline IHC score & 319 & 3.75 & - & 3.05 & & 0.089 (CXCL16) \\
\hline & & & & & & 0.087 (CXCR6) \\
\hline
\end{tabular}

IHC, immunohistochemical staining; BCR, biochemical serum PSA recurrence; PSA; prostate-specific antigen, LN; lymph node. p-values were obtained from the IHC score using the Chi-square test.

(62.9\%), and CXCR6 expression was observed in 18 of 35 samples (51.4\%) (Fig. 2).

The mean IHC score for CXCL16 and CXCR6 in the PCa tissues was 3.75 and 3.05, respectively. The intensity/ percentage scores for CXCL16 and CXCR6 were 2.16/1.58 and 1.15/1.89, respectively. The mean IHC score for CXCL16 and CXCR6 was lower in patients with $\mathrm{BPH}$ than in the $\mathrm{PCa}$ tissues (2.01 and $1.98, \mathrm{p}=0.038$ and $\mathrm{p}=0.042$, respectively). The intensity/percentage scores for CXCL16 and CXCR6 in BPH were 1.05/0.95 and 0.82/1.16, respectively.
Quantitative real-time $R T-P C R$

In vitro. Expression of CXCL16 and CXCR6 mRNA was stronger in the two PCa cell lines than in the PrEC cell line (Fig. 3A).

In vivo. Expression of CXCL16 and CXCR6 mRNA was stronger in patients with PCa compared to those with BPH. Additionally, CXCL16 and CXCR6 mRNA was weakly expressed in the normal bone, lung and liver tissues, whereas the expression of CXCL16 was strong in the metastatic bone tissues (Fig. 3B). 
Correlation between clinicopathological features and the chemokine receptor. As shown in Table I, CXCL16 immunoreactivity increased in conjuction with Gleason score, T stage, tumor volume, perineural invasion and lymph node metastasis. The respective IHC scores for CXCL16 were 4.81 and 2.65 for high (>7) and low ( $\leq 7)$ Gleason scores, 4.58 and 2.87 for high $(>\mathrm{T} 2 \mathrm{c})$ and low ( $\leq \mathrm{T} 2 \mathrm{c})$ pathological T stage, 5.15 and $2.30 \mathrm{in}$ positive and negative perineural invasion, and 5.08 and 2.37 in positive and negative lymph node metastasis, respectively. Serum PSA biochemical recurrence was not related to the CXCL16 IHC score, but was related to the preoperative PSA, Gleason score and pathological $\mathrm{T}$ stage.

The expression of CXCR6 was weakly correlated with the Gleason score $(p=0.051)$, but not with other clinicopathological parameters, although it was related to the expression of CXCL16. The IHC scores for CXCR6 were 3.48 and 2.57 for high (>7) and low ( $\leq 7)$ Gleason scores. Similar to CXCL16, serum PSA biochemical recurrence was not correlated with the CXCR6 IHC score.

In a multivariate Cox regression analysis of biochemical serum PSA recurrence, although the serum PSA and T stage were significantly related to biochemical recurrence, the expression levels of CXCL16 and CXCR6 were not independent factors ( $\mathrm{p}=0.157$ and $\mathrm{p}=0.882$, respectively).

\section{Discussion}

Chemokines are small secreted peptides that control the adhesion and transendothelial migration of leukocytes, lymphocytes and monocytes, particularly during immune and inflammatory reactions (11). Chemokines and their receptors also play significant roles in tumor metastasis, recurrence and angiogenesis (12). There are numerous reports on the implications of the expression of CXCL12/CXCR4 in PCa. However, few studies have examined the association between the expression of CXCL16/CXCR6 and clinical features in patients with PCa. We compared the expression of CXCL16/CXCR6 in patients with $\mathrm{PCa}$ according to clinical features, including serum PSA, Gleason score, stage and other pathological parameters.

The expression of the chemokine and its receptor (CXCL16/CXCR6) was examined in PCa using IHC staining and real-time RT-PCR. Chemokine receptors are important in the metastatic behavior of solid cancers (11). There are conflicting reports that the expression of the chemokine, but not its receptor (13), or of both (14), is present in PCa. Mochizuki et al (13) reported that the expression of CXCR4, but not its ligand, stromal derived factor-1 (SDF-1), was increased in $\mathrm{PCa}$; this indicates that $\mathrm{PCa}$ cells are affected not only by endogenous, but also by exogenous, SDF-1. However, we found that the expression of both CXCR6 and CXCL16 was increased to a greater extent in PCa cell lines and tissues compared to PrEC or BPH tissues. The expression of CXCL16/CXCR6 was stronger in the PC-3 and LNCaP cells than in PrEC cells in vitro. The expression of CXCL16/ CXCR6 was also stronger in tissues from patients with $\mathrm{PCa}$ than in those from patients with non-malignant prostate disease in vivo. These results suggest that CXCL16/CXCR6 is expressed de novo during prostate tumorigenesis.

Koizumi et al (3) suggested that high CXCR4 expression predicted a poor prognosis in melanoma and lung, pancreatic, ovarian, colorectal and breast cancer. Li et al (15) observed CXCR4 expression in approximately $75 \%$ of biopsy specimens of invasive ductal carcinoma, and found that high CXCR4 expression was correlated with the decreased overall survival of patients with breast cancer. Zhang et al (11) reported that CXCL12 and its receptor CXCR4 are related to PCa perineural invasion. Other authors have suggested that the chemokine and its receptor mark cancer arising in an inflammatory milieu, and mediate the pro-tumorigenic effects of inflammation through direct effects on cancer cell growth and by inducing the migration and proliferation of tumorassociated leukocytes (14). In the present study, the expression of CXCL16 and CXCR6 was also associated with Gleason grade, indicating that aggressive PCa expresses CXCR6 more strongly. We also found that the expression of CXCL16 was increased in patients with perineural invasion, lymph node metastasis, large tumor volume and high pathological T stage, suggesting that it is associated with local invasion or distant metastasis. The correlation between the expression of CXCR6 and clinicopathological parameters was weak. We believe that this may have been due to the relatively weak intensity of CXCR6 upon IHC staining compared to that of CXCL16.

The high mortality rate in patients with $\mathrm{PCa}$ is attributable to the spread of malignant cells to many tissues; the majority of men with progressive PCa develop bone metastases (16). There is a growing interest in the early detection and screening of men for $\mathrm{PCa}$, and also for a greater understanding of the mechanisms that lead to metastasis (17). Tumor cells invade the skeleton via interaction between tumor cells and the bone microenvironment, which consists of stromal cells, osteoblasts, osteoclasts, endothelial cells, fibroblasts, adipocytes, bone marrow precursor cells, cells of the immune system and the extracellular matrix. The growth factors and chemokines produced in the bone microenvironment enhance the growth of PCa cells and cancer progression in bones (18).

While the sequence of cancer metastasis is well recognized, little is known about the molecular mechanism(s) regulating it (19). However, it has been demonstrated that sites of metastasis are not random. It appears that metastasis is dependent on the properties of cancer cells and the microenvironment of specific organ sites (20). While these preferences are difficult to explain or predict, evidence increasingly suggests that they are the result of interactions between chemokines and their receptors (21). Bone metastasis is more frequent in PCa than lung or liver metastasis, which are common metastatic sites for other cancers. Muller et al (22) reported that CXCR4 was highly expressed in human breast cancer cells, and that the highest receptor expression was observed in those organs that are usually the primary sites of breast cancer metastasis (22). Koizumi et al (3) also suggested that the expression of CXCR4 in biopsy specimens from primary gastric tumors may aid in the preoperative evaluation of the risk of recurrence of peritoneal carcinomatosis. Additionally, $\mathrm{Hu}$ et al (8) suggested that CXCL12 and CXCR4 are crucial factors involved in PCa bone metastasis. Thus, we hypothesized that chemokines and their receptors help to define the bone metastasis of PCa. To examine this, we evaluated the expression of the chemokine CXCL16 in bone marrow, lung and liver specimens. The human bone marrow tissues exhibited similar expression of CXCL16 compared to the lung or liver samples. However, the 
expression of CXCL16 was significantly increased in the metastatic bone tissues. This suggests that CXCR6 and its ligand CXCL16 influence the metastatic pattern of each tumor, and that the evaluation of other factor(s) related to CXCL16 levels in the metastatic bone marrow of patients with advanced $\mathrm{PCa}$ may aid in predicting the risk of bone metastasis.

\section{Acknowledgements}

This study was supported by a Medical Research Institute and Busan Cancer Center Grant (2010-31) from Pusan National University Hospital.

\section{References}

1. Ha HK, Yun CJ, Lee SS, et al: Survival rates and related factors in men with hormone-refractory prostate cancer. Korean J Urol 50: 649-655, 2009.

2. Wang J, Lu Y, Koch AE, Zhang J and Taichman RS: CXCR6 induces prostate cancer progression by the AKT/mammalian target of rapamycin signaling pathway. Cancer Res 68: 10367-10376, 2008.

3. Koizumi K, Hojo S,Akashi T, Yasumoto K and Saiki I: Chemokine receptors in cancer metastasis and cancer cell-derived chemokines in host immune response. Cancer Sci 98: 1652-1658, 2007.

4. Hojo S, Koizumi K, Tsuneyama K, et al: High-level expression of chemokine CXCL16 by tumor cells correlates with a good prognosis and increased tumor-infiltrating lymphocytes in colorectal cancer. Cancer Res 67: 4725-4731, 2007.

5. Ludwig A, Schulte A, Schnack C, et al: Enhanced expression and shedding of the transmembrane chemokine CXCL16 by reactive astrocytes and glioma cells. J Neurochem 93: 1293-1303, 2005.

6. Scholz F, Schulte A, Adamski F, et al: Constitutive expression and regulated release of the transmembrane chemokine CXCL16 in human and murine skin. J Invest Dermatol 127: 1444-1455, 2007.

7. Tabata S, Kadowaki N, Kitawaki T, et al: Distribution and kinetics of SR-PSOX/CXCL16 and CXCR6 expression on human dendritic cell subsets and CD4+ ${ }^{+}$cells. J Leukoc Biol 77: 777-786, 2005.

8. Hu W, Zhen X, Xiong B, Wang B, Zhang W and Zhou W: CXCR6 is expressed in human prostate cancer in vivo and is involved in the in vitro invasion of PC3 and LNCap cells. Cancer Sci 99: 1362-1369, 2008.
9. Sobin LH and Fleming ID: TNM Classification of Malignant Tumors, 5th edition (1997). Union Internationale Contre le Cancer and the American Joint Committee on Cancer. Cancer 80: 1803-1804, 1997.

10. Epstein JI, Allsbrook WC Jr., Amin MB and Egevad LL: The 2005 International Society of Urological Pathology (ISUP) Consensus Conference on Gleason Grading of Prostatic Carcinoma. Am J Surg Pathol 29: 1228-1242, 2005.

11. Zhang S, Qi L, Li M, et al: Chemokine CXCL12 and its receptor CXCR4 expression are associated with perineural invasion of prostate cancer. J Exp Clin Cancer Res 27: 62, 2008.

12. Balkwill F: The significance of cancer cell expression of the chemokine receptor CXCR4. Semin Cancer Biol 14: 171-179, 2004.

13. Mochizuki H, Matsubara A, Teishima J, et al: Interaction of ligand-receptor system between stromal-cell-derived factor-1 and CXC chemokine receptor 4 in human prostate cancer: a possible predictor of metastasis. Biochem Biophys Res Commun 320: 656-663, 2004.

14. Darash-Yahana M, Gillespie JW, Hewitt SM, et al: The chemokine CXCL16 and its receptor, CXCR6, as markers and promoters of inflammation-associated cancers. PLoS One 4: E6695, 2009.

15. Li YM, Pan Y, Wei Y, et al: Upregulation of CXCR4 is essential for HER2-mediated tumor metastasis. Cancer Cell 6: 459-469, 2004.

16. Jacobs SC: Spread of prostatic cancer to bone. Urology 21: 337-344, 1983

17. Taichman RS, Cooper C, Keller ET, Pienta KJ, Taichman NS and McCauley LK: Use of the stromal cell-derived factor-1/CXCR4 pathway in prostate cancer metastasis to bone. Cancer Res 62: 1832-1837, 2002.

18. Lu Y, Wang J, Xu Y, et al: CXCL16 functions as a novel chemotactic factor for prostate cancer cells in vitro. Mol Cancer Res 6: 546-554, 2008.

19. Al-Mehdi AB, Tozawa K, Fisher AB, Shientag L, Lee A and Muschel RJ: Intravascular origin of metastasis from the proliferation of endothelium-attached tumor cells: a new model for metastasis. Nat Med 6: 100-102, 2000.

20. Hart IR and Fidler IJ: Role of organ selectivity in the determination of metastatic patterns of B16 melanoma. Cancer Res 40: 2281-2287, 1980.

21. Zlotnik A: Chemokines and cancer. Int J Cancer 119: 2026-2029, 2006.

22. Muller A, Homey B, Soto H, et al: Involvement of chemokine receptors in breast cancer metastasis. Nature 410: 50-56, 2001. 